VOLUME 30 (2021) 304-311

DOI: $10.24330 /$ ieja.969942

\title{
SOME NEW CHARACTERIZATIONS OF PARTIAL ISOMETRIES IN RINGS WITH INVOLUTION
}

\author{
Dandan Zhao and Junchao Wei \\ Received: 12 October 2020; Accepted: 25 January 2021 \\ Communicated by Abdullah Harmancı
}

\begin{abstract}
In this paper, some characterizations of partial isometries, normal elements and strongly EP elements are given by the construction of EP elements. At the same time, the partial isometry elements are characterized by the existence of solutions of equations in rings in a given set, and also by the general form of solutions of given equations.
\end{abstract}

Mathematics Subject Classification (2020): 15A09; 16U99; 16W10

Keywords: Group inverse, MP-invertible element, partial isometry element, $E P$ element, strongly EP element, normal element

\section{Introduction}

Let $R$ be an associative ring with 1 . An element $a \in R$ is said to be group invertible if there exists $a^{\#} \in R$ such that

$$
a a^{\#} a=a, \quad a^{\#} a a^{\#}=a^{\#}, \quad a a^{\#}=a^{\#} a .
$$

The element $a^{\#}$ is called the group inverse of $a$, which is uniquely determined by the above equations [1].

An involution $*: a \longmapsto a^{*}$ in a ring $R$ is an anti-isomorphism of degree 2 , that is

$$
\left(a^{*}\right)^{*}=a, \quad(a+b)^{*}=a^{*}+b^{*}, \quad(a b)^{*}=b^{*} a^{*} .
$$

An element $a \in R$ satisfying $a^{*} a=a a^{*}$ is called normal [4].

An element $a^{+}$in $R$ is called the Moore-Penrose inverse (MP-inverse) of $a$, if

$$
a a^{+} a=a, \quad a^{+} a a^{+}=a^{+}, \quad\left(a a^{+}\right)^{*}=a a^{+}, \quad\left(a^{+} a\right)^{*}=a^{+} a .
$$

If such $a^{+}$exists, then it is unique [3]. Denote by $R^{\#}$ and $R^{+}$the set of all group invertible elements of $R$ and the set of all MP-invertible elements of $R$.

An element $a$ is said to be $E P$ if $a \in R^{\#} \cap R^{+}$and satisfies $a^{\#}=a^{+}$. We denote by $R^{E P}$ the set of all $E P$ elements of $R$.

Project supported by the Foundation of Natural Science of China (10771282). 
An element $a$ is called a partial isometry if $a \in R^{+}$and $a a^{*} a=a$. And $a$ is said to be strongly $E P$ if $a \in R^{E P}$ is a partial isometry. We denote the set of all partial isometry elements and strongly $E P$ elements of $R$ by $R^{P I}$ and $R^{S E P}$, respectively.

There are many studies on $E P$ element, $P I$ element and strongly $E P$ element, see for example $[2,4,5,6,7,8,9,10]$.

In [11], EP elements are characterized by constructing the existence of solutions of appropriate equations in a given set, which is a new method to study generalized inverse in rings.

In this paper, some characterizations of partial isometries, normal elements and strongly EP elements are given by the construction of EP elements. At the same time, the partial isometry elements are characterized by the existence of solutions of equations in rings in a given set, and also by the general form of solutions of given equations.

\section{Main Results}

Lemma 2.1. Let $a \in R^{\#} \cap R^{+}$and $x \in R$.

(1) If $a^{+} a^{+} x=0$, then $a^{+} x=0$;

(2) If $x a^{+} a^{+}=0$, then $x a^{+}=0$.

Proof. (1) Since $a^{+} a^{+} x=0, a^{*} a^{+} x=\left(a^{*} a a^{+}\right) a^{+} x=a^{*} a\left(a^{+} a^{+} x\right)=0$. Noting that $a^{+}=\left(a^{+} a\right)^{*} a^{+}=\left(a^{+} a a^{\#} a\right)^{*} a^{+}=\left(a^{\#}\right)^{*} a^{*} a^{+} a a^{+}=\left(a^{\#}\right)^{*} a^{*} a^{+}$, we have $a^{+} x=\left(a^{\#}\right)^{*} a^{*} a^{+} x=0$.

Similarly, we can show (2).

It is well known that for $a \in R^{+}, a \in R^{P I}$ if and only if $a^{+}=a^{*}$. Hence we have the following Lemma.

Lemma 2.2. Let $a \in R^{\#} \cap R^{+}$. Then $a \in R^{P I}$ if and only if the equation

$$
x\left(a^{+}-a^{*}\right)=0
$$

has at least one solution in $\chi_{a}=\left\{a, a^{\#}, a^{+}, a^{*},\left(a^{\#}\right)^{*},\left(a^{+}\right)^{*}\right\}$.

Proof. " $\Rightarrow$ " If $a \in R^{P I}$, then $a^{+}=a^{*}$. This infers every element of $\chi_{a}$ is a solution of the equation (1).

" $\Leftarrow "$ (1) If $x=a$ is a solution, then $a\left(a^{+}-a^{*}\right)=0$, that is $a a^{+}=a a^{*}$. Hence $a \in R^{P I}$ by [5, Theorem 2.1].

(2) If $x=a^{\#}$ is a solution, then $a^{\#}\left(a^{+}-a^{*}\right)=0$. Pre-multiplying the equality by $a^{2}$, we have $a\left(a^{+}-a^{*}\right)=0$. Hence $a \in R^{P I}$ by (1).

(3) If $x=a^{+}$is a solution, then $a^{+}\left(a^{+}-a^{*}\right)=0$, this gives $a^{+} a^{+}\left(a a^{+}-a a^{*}\right)=0$. By Lemma 2.1, we have $a^{+}\left(a a^{+}-a a^{*}\right)=0$, this is $a^{+}=a^{*}$. Hence $a \in R^{P I}$. 
(4) If $x=a^{*}$ is a solution, then $a^{*}\left(a^{+}-a^{*}\right)=0$. Pre-multiplying the equality by $a^{+}\left(a^{+}\right)^{*}$, yielding $a^{+}\left(a^{+}-a^{*}\right)=0$. Hence $a \in R^{P I}$ by $(3)$.

(5) If $x=\left(a^{\#}\right)^{*}$ is a solution, then $\left(a^{\#}\right)^{*}\left(a^{+}-a^{*}\right)=0$. Pre-multiplying the equality by $\left(a^{*}\right)^{2}$, we have $a^{*}\left(a^{+}-a^{*}\right)=0$. Hence $a \in R^{P I}$ by (4).

(6) If $x=\left(a^{+}\right)^{*}$ is a solution, then $\left(a^{+}\right)^{*}\left(a^{+}-a^{*}\right)=0$. Pre-multiplying the equality by $a a^{*}$, one gets $a\left(a^{+}-a^{*}\right)=0$. Hence $a \in R^{P I}$ by (1).

Similarly, we have the following Lemma.

Lemma 2.3. Let $a \in R^{\#} \cap R^{+}$. Then $a \in R^{P I}$ if and only if the equation

$$
\left(a^{+}-a^{*}\right) x=0
$$

has at least one solution in $\chi_{a}$.

Theorem 2.4. Let $a \in R^{\#} \cap R^{+}$. Then $a \in R^{P I}$ if and only if the equation

$$
x\left(a^{+}-a^{*}\right) y=0
$$

has at least one solution in $\chi_{a}^{2}=\left\{(x, y) \mid x, y \in \chi_{a}\right\}$.

Proof. " $\Rightarrow$ " It is an immediate result of Lemma 2.2.

$" \Leftarrow "$ (1) If $y=a$, we have the following equation

$$
x\left(a^{+}-a^{*}\right) a=0 .
$$

Post-multiplying the equation (4) by $a^{+}$, one gets $x\left(a^{+}-a^{*}\right)=0$. Hence $a \in R^{P I}$ by Lemma 2.2 .

(2) If $y=a^{\#}$, then we have the following equation

$$
x\left(a^{+}-a^{*}\right) a^{\#}=0 .
$$

Post-multiplying the equation (5) by $a^{2}$, one has $x\left(a^{+}-a^{*}\right) a=0$. Hence $a \in R^{P I}$ by (1).

(3) If $y=a^{+}$, then we obtain the following equation

$$
x\left(a^{+}-a^{*}\right) a^{+}=0 .
$$

So

$$
x\left(a^{+} a-a^{*} a\right) a^{+} a^{+}=0 .
$$

By Lemma $2.1 x\left(a^{+}-a^{*}\right)=0$. Hence $a \in R^{P I}$ by Lemma 2.2 .

(4) If $y=a^{*}$, then we have the following equation

$$
x\left(a^{+}-a^{*}\right) a^{*}=0 .
$$

Post-multiplying the equation (7) by $\left(a^{+}\right)^{*} a^{+}$, one gets $x\left(a^{+}-a^{*}\right) a^{+}=0$. Hence $a \in R^{P I}$ by (3). 
(5) If $y=\left(a^{\#}\right)^{*}$, then we have the following equation

$$
x\left(a^{+}-a^{*}\right)\left(a^{\#}\right)^{*}=0 .
$$

Post-multiplying the equation (8) by $\left(a^{*}\right)^{2}$, we have $x\left(a^{+}-a^{*}\right) a^{*}=0$. Hence $a \in R^{P I}$ by (4).

(6) If $y=\left(a^{+}\right)^{*}$, then we have the following equation

$$
x\left(a^{+}-a^{*}\right)\left(a^{+}\right)^{*}=0 .
$$

Post-multiplying the equation (9) by $a^{*} a$, we have $x\left(a^{+}-a^{*}\right) a=0$. Hence $a \in R^{P I}$ by (1).

Remark 2.5. Equation (1) can be generalized to

$$
x a^{+}-y a^{*}=0 .
$$

It is easy to prove that the general solution of the equation (10) is given by

$$
\left\{\begin{array}{l}
x=-p a+u-u a^{+} a \\
y=-p\left(a^{+}\right)^{*}+v-v a^{+} a
\end{array}, \text { where } p, u, v \in R .\right.
$$

Lemma 2.6. Let $a \in R^{\#} \cap R^{+}$. Then $a \in R^{P I}$ if and only if $a^{+}=a^{+}\left(a^{+}\right)^{*} a^{+}$.

Proof. " $\Rightarrow$ " Assume that $a \in R^{P I}$, then $\left(a^{+}\right)^{*}=a$. Hence $a^{+}=a^{+}\left(a^{+}\right)^{*} a^{+}$. " $\Leftarrow$ " Suppose that $a^{+}=a^{+}\left(a^{+}\right)^{*} a^{+}$, then $a=a a^{+} a=a a^{+}\left(a^{+}\right)^{*} a^{+} a=\left(a^{+}\right)^{*}$. Thus $a \in R^{P I}$.

Proposition 2.7. Let $a \in R^{\#} \cap R^{+}$. Then $a \in R^{P I}$ if and only if the general solution of the equation (10) is given by

$$
\left\{\begin{array}{l}
x=-p\left(a^{+}\right)^{*}+u-u a^{+} a \\
y=-p\left(a^{+}\right)^{*}+v-v a^{+} a
\end{array}, \text { where } p, u, v \in R .\right.
$$

Proof. " $\Rightarrow$ " This is an immediate corollary of the formula (11).

" $\Leftarrow$ " If the general solution of the equation (10) is given by the formula (12), then $\left(-p\left(a^{+}\right)^{*}+u-u a^{+} a\right) a^{+}-\left(-p\left(a^{+}\right)^{*}+v-v a^{+} a\right) a^{*}=0$, that is $p\left(a^{+}\right)^{*} a^{+}=p a a^{+}$ for any $p \in R$. Especially, choose $p=a^{+}$, one yields $a^{+}\left(a^{+}\right)^{*} a^{+}=a^{+} a a^{+}=a^{+}$. Hence $a \in R^{P I}$ by Lemma 2.6.

Similarly, we have the following Proposition. 
Proposition 2.8. Let $a \in R^{\#} \cap R^{+}$. Then $a \in R^{P I}$ if and only if the general solution of the equation (10) is given by

$$
\left\{\begin{array}{l}
x=-p a+u-u a^{+} a \\
y=-p a+v-v a^{+} a
\end{array}, \text { where } p, u, v \in R .\right.
$$

Lemma 2.9. Let $a \in R^{\#} \cap R^{+}$. Then $\left(a^{+}\right)^{*} a^{+} a^{*} a a^{*} \in R^{E P}$ with

$$
\left(\left(a^{+}\right)^{*} a^{+} a^{*} a a^{*}\right)^{+}=\left(a^{+}\right)^{*} a^{+}\left(a^{\#}\right)^{*} a a^{*} .
$$

Proof. Since

$$
\begin{aligned}
\left(\left(a^{+}\right)^{*} a^{+} a^{*} a a^{*}\right)\left(\left(a^{+}\right)^{*} a^{+}\left(a^{\#}\right)^{*} a a^{*}\right) & =\left(a^{+}\right)^{*} a^{+} a^{*}\left(a a^{*}\left(a^{+}\right)^{*}\right) a^{+}\left(a^{\#}\right)^{*} a a^{*} \\
& =\left(a^{+}\right)^{*} a^{+}\left(a^{*} a a^{+}\right)\left(a^{\#}\right)^{*} a a^{*} \\
& =\left(a^{+}\right)^{*} a^{+} a^{*}\left(a^{\#}\right)^{*} a a^{*} \\
& =\left(a^{+}\right)^{*} a^{+} a a^{*} \\
& =\left(a^{+}\right)^{*} a^{*} \\
& =\left(a a^{+}\right)^{*} \\
& =a a^{+}, \\
\left(\left(a^{+}\right)^{*} a^{+}\left(a^{\#}\right)^{*} a a^{*}\right)\left(\left(a^{+}\right)^{*} a^{+} a^{*} a a^{*}\right) & =\left(a^{+}\right)^{*} a^{+}\left(a^{\#}\right)^{*}\left(a a^{*}\left(a^{+}\right)^{*}\right) a^{+} a^{*} a a^{*} \\
& =\left(a^{+}\right)^{*} a^{+}\left(\left(a^{\#}\right)^{*} a a^{+}\right) a^{*} a a^{*} \\
& =\left(a^{+}\right)^{*}\left(a^{+}\left(a^{\#}\right)^{*} a^{*}\right) a a^{*} \\
& =\left(a^{+}\right)^{*} a^{+} a a^{*} \\
& =a a^{+} .
\end{aligned}
$$

Hence $\left(a^{+}\right)^{*} a^{+} a^{*} a a^{*} \in R^{E P}$ with $\left(\left(a^{+}\right)^{*} a^{+} a^{*} a a^{*}\right)^{+}=\left(a^{+}\right)^{*} a^{+}\left(a^{\#}\right)^{*} a a^{*}$.

Proposition 2.10. Let $a \in R^{\#} \cap R^{+}$. Then

(1) $a \in R^{P I}$ if and only if $\left(\left(a^{+}\right)^{*} a^{+} a^{*} a a^{*}\right)^{+}=a a^{+}\left(a^{\#}\right)^{*} a a^{*}$;

(2) $a \in R^{P I}$ if and only if $\left(\left(a^{+}\right)^{*} a^{+} a^{*} a a^{*}\right)^{+}=\left(a^{+}\right)^{*} a^{+}\left(a^{\#}\right)^{*}$;

(3) $a$ is normal if and only if $\left(\left(a^{+}\right)^{*} a^{+} a^{*} a a^{*}\right)^{+}=\left(a^{+}\right)^{*}$;

(4) $a \in R^{S E P}$ if and only if $\left(\left(a^{+}\right)^{*} a^{+} a^{*} a a^{*}\right)^{+}=\left(a^{+}\right)^{*} a^{+}\left(a^{+}\right)^{*}$.

Proof. (1) " $\Rightarrow$ " Since $a \in R^{P I},\left(a^{+}\right)^{*}=a$. Hence, by Lemma 2.9, we have

$$
\left(\left(a^{+}\right)^{*} a^{+} a^{*} a a^{*}\right)^{+}=\left(a^{+}\right)^{*} a^{+}\left(a^{\#}\right)^{*} a a^{*}=a a^{+}\left(a^{\#}\right)^{*} a a^{*} .
$$

$" \Leftarrow "$ Assume that $\left(\left(a^{+}\right)^{*} a^{+} a^{*} a a^{*}\right)^{+}=a a^{+}\left(a^{\#}\right)^{*} a a^{*}$, then $\left(a^{+}\right)^{*} a^{+}\left(a^{\#}\right)^{*} a a^{*}=$ $a a^{+}\left(a^{\#}\right)^{*} a a^{*}$ by Lemma 2.9. Post-multiplying the equality by $\left(a^{+}\right)^{*} a^{+}$, one yields 
$\left(a^{+}\right)^{*} a^{+}\left(a^{\#}\right)^{*}=a a^{+}\left(a^{\#}\right)^{*}$. Pre-multiplying the equality by $a^{*}$, one has $a^{+}\left(a^{\#}\right)^{*}=$ $a^{*}\left(a^{\#}\right)^{*}$, this gives $a^{+}=a^{+}\left(a^{\#}\right)^{*} a^{*}=a^{*}\left(a^{\#}\right)^{*} a^{*}=a^{*}$. Thus $a \in R^{P I}$.

$(2)$ " $\Rightarrow$ " Since $a \in R^{P I}, a^{*}=a^{+}$. This infers $\left(a^{\#}\right)^{*} a a^{*}=\left(a^{\#}\right)^{*} a a^{+}=\left(a^{\#}\right)^{*}$. Hence $\left(\left(a^{+}\right)^{*} a^{+} a^{*} a a^{*}\right)^{+}=\left(a^{+}\right)^{*} a^{+}\left(a^{\#}\right)^{*}$ by Lemma 2.9 .

" $\Leftarrow$ " Assume that $\left(\left(a^{+}\right)^{*} a^{+} a^{*} a a^{*}\right)^{+}=\left(a^{+}\right)^{*} a^{+}\left(a^{\#}\right)^{*}$, then $\left(a^{+}\right)^{*} a^{+}\left(a^{\#}\right)^{*} a a^{*}=$ $\left(a^{+}\right)^{*} a^{+}\left(a^{\#}\right)^{*}$ by Lemma 2.9. Pre-multiplying by $a^{*}$, we have $a^{+}\left(a^{\#}\right)^{*} a a^{*}=$ $a^{+}\left(a^{\#}\right)^{*}$. Again pre-multiplying by $\left(a^{\#}\right)^{*} a^{*} a$, one obtains $\left(a^{\#}\right)^{*} a a^{*}=\left(a^{\#}\right)^{*}$, this gives $a^{\#}=a a^{*} a^{\#}$. Hence $a \in R^{P I}$.

$(3)$ " $\Rightarrow$ " Since $a$ is normal, $a a^{*}=a^{*} a$ and $a \in R^{E P}$. Hence, by Lemma 2.9, we obtain

$$
\begin{aligned}
\left(\left(a^{+}\right)^{*} a^{+} a^{*} a a^{*}\right)^{+} & =\left(a^{+}\right)^{*} a^{+}\left(a^{\#}\right)^{*} a a^{*} \\
& =\left(a^{+}\right)^{*} a^{+}\left(a^{+}\right)^{*} a^{*} a \\
& =\left(a^{+}\right)^{*} a^{+} a \\
& =\left(a^{+}\right)^{*} .
\end{aligned}
$$

" $\Leftarrow "$ Assume that $\left(\left(a^{+}\right)^{*} a^{+} a^{*} a a^{*}\right)^{+}=\left(a^{+}\right)^{*}=\left(a^{*}\right)^{+}$, then $\left(a^{+}\right)^{*} a^{+} a^{*} a a^{*}=a^{*}$. Post-multiplying the equality by $\left(a^{+}\right)^{*} a^{+}$, one gets $\left(a^{+}\right)^{*} a^{+} a^{*}=a^{+}$, this gives $a a^{+} a^{+}=a a^{+}\left(a^{+}\right)^{*} a^{+} a^{*}=\left(a^{+}\right)^{*} a^{+} a^{*}=a^{+}$. Hence $a \in R^{E P}$. Now $a^{*} a^{\#}=$ $a^{*} a^{+}=a^{*}\left(a^{+}\right)^{*} a^{+} a^{*}=a^{+} a^{*}=a^{\#} a^{*}$. Thus $a$ is normal.

(4) " $\Rightarrow$ " It is an immediate result of Lemma 2.9, because $a^{+}=a^{\#}$ and $a \in R^{P I}$. " $\Leftarrow$ " Assume that $\left(\left(a^{+}\right)^{*} a^{+} a^{*} a a^{*}\right)^{+}=\left(a^{+}\right)^{*} a^{+}\left(a^{+}\right)^{*}$, then by Lemma 2.9, one has $\left(a^{+}\right)^{*} a^{+}\left(a^{\#}\right)^{*} a a^{*}=\left(a^{+}\right)^{*} a^{+}\left(a^{+}\right)^{*}$. Post-multiplying the equality by $1-a a^{+}$, one yields $\left(a^{+}\right)^{*} a^{+}\left(a^{+}\right)^{*}\left(1-a a^{+}\right)=0$. Pre-multiplying the equality by $a^{*} a a^{*}$, one has $a^{+} a\left(1-a a^{+}\right)=0$, this gives $a\left(1-a a^{+}\right)=0$. Hence $a \in R^{E P}$. It follows that $\left(\left(a^{+}\right)^{*} a^{+}\left(a^{\#}\right)^{*} a a^{*}\right)^{+}=\left(a^{+}\right)^{*} a^{+}\left(a^{+}\right)^{*}=\left(a^{+}\right)^{*} a^{+}\left(a^{\#}\right)^{*}$. Hence $a \in R^{P I}$ by (2). Therefore $a \in R^{S E P}$.

Lemma 2.11. Let $a \in R^{\#} \cap R^{+}$. Then $a a^{+} a^{*} a a^{*} \in R^{E P}$ with

$$
\left(a a^{+} a^{*} a a^{*}\right)^{+}=\left(a^{+}\right)^{*} a^{+}\left(a^{\#}\right)^{*} .
$$

Proof.

$$
\begin{aligned}
\left(a a^{+} a^{*} a a^{*}\right)\left(\left(a^{+}\right)^{*} a^{+}\left(a^{\#}\right)^{*}\right) & =a a^{+} a^{*} a a^{+}\left(a^{\#}\right)^{*} \\
& =a a^{+} a^{*}\left(a^{\#}\right)^{*} \\
& =a a^{+}
\end{aligned}
$$




$$
\begin{aligned}
\left(\left(a^{+}\right)^{*} a^{+}\left(a^{\#}\right)^{*}\right)\left(a a^{+} a^{*} a a^{*}\right) & =\left(a^{+}\right)^{*} a^{+}\left(a^{\#}\right)^{*} a^{*} a a^{*} \\
& =\left(a^{+}\right)^{*} a^{+} a a^{*} \\
& =a a^{+} .
\end{aligned}
$$

Hence $a a^{+} a^{*} a a^{*} \in R^{E P}$ with $\left(a a^{+} a^{*} a a^{*}\right)^{+}=\left(a^{+}\right)^{*} a^{+}\left(a^{\#}\right)^{*}$.

Proposition 2.12. Let $a \in R^{\#} \cap R^{+}$. Then

(1) $a \in R^{P I}$ if and only if $\left(\left(a^{+}\right)^{*} a^{+}\left(a^{\#}\right)^{*}\right)^{+}=a a^{+} a^{*}$;

(2) $a \in R^{E P}$ if and only if $\left(\left(a^{+}\right)^{*} a^{+}\left(a^{\#}\right)^{*}\right)^{+}=a^{*} a a^{*}$;

(3) $a \in R^{S E P}$ if and only if $\left(\left(a^{+}\right)^{*} a^{+}\left(a^{\#}\right)^{*}\right)^{+}=a^{*}$.

Proof. (1) " $\Rightarrow$ " Since $a \in R^{P I}, a^{*}=a^{+}$. Hence, by Lemma 2.11, we get

$$
\left(\left(a^{+}\right)^{*} a^{+}\left(a^{\#}\right)^{*}\right)^{+}=a a^{+} a^{*} a a^{*}=a a^{+} a^{*} a a^{+}=a a^{+} a^{*} .
$$

" $\Leftarrow$ " Assume that $\left(\left(a^{+}\right)^{*} a^{+}\left(a^{\#}\right)^{*}\right)^{+}=a a^{+} a^{*}$, then $a a^{+} a^{*} a a^{*}=a a^{+} a^{*}$ by Lemma 2.11. Pre-multiplying the equality by $\left(a^{\#}\right)^{*} a^{*}$, one yields $a^{*} a a^{*}=a^{*}$, this gives $a=a a^{*} a$. Hence $a \in R^{P I}$.

(2) " $\Rightarrow$ " Since $a \in R^{E P}, a a^{+}=a^{+} a$. Hence

$$
\left(\left(a^{+}\right)^{*} a^{+}\left(a^{\#}\right)^{*}\right)^{+}=a a^{+} a^{*} a a^{*}=a^{+} a a^{*} a a^{*}=a^{*} a a^{*} .
$$

" $\Leftarrow$ " Assume that $\left(\left(a^{+}\right)^{*} a^{+}\left(a^{\#}\right)^{*}\right)^{+}=a^{*} a a^{*}$. Then $a a^{+} a^{*} a a^{*}=a^{*} a a^{*}$ by Lemma 2.11. Post-multiplying the equality by $\left(a^{+}\right)^{*} a^{+}\left(a^{+}\right)^{*}$, one gets $a a^{+} a^{+} a=a^{+} a$. Hence $a \in R^{E P}$.

(3) " $\Rightarrow$ " Since $a \in R^{S E P}, a \in R^{E P}$ and $a^{*}=a^{+}$. By $(2)\left(\left(a^{+}\right)^{*} a^{+}\left(a^{\#}\right)^{*}\right)^{+}=$ $a^{*} a a^{*}=a^{*} a a^{+}=a^{*}$.

" $\Leftarrow "$ Assume that $\left(\left(a^{+}\right)^{*} a^{+}\left(a^{\#}\right)^{*}\right)^{+}=a^{*}$, then $a a^{+} a^{*} a a^{*}=a^{*}$ by Lemma 2.11. Post-multiplying the equality by $\left(a^{+}\right)^{*} a^{+}$, one obtains $a a^{+} a^{*}=a^{+}$. Hence $a \in$ $R^{S E P}$ by [1, Theorem 2.3].

Acknowledgement. The authors would like to thank the referee for the valuable suggestions and comments.

\section{References}

[1] A. Ben-Israel and T. N. E Greville, Generalized Inverses: Theory and Applications, 2nd. ed., Springer, New York, 2003.

[2] S. Karanasios, EP elements in rings and semigroup with involution and $C^{*}$ algebras, Serdica Math. J., 41 (2015), 83-116.

[3] J. J. Koliha and P. Patricio, Elements of rings with equal spectral idempotents, J. Aust. Math. Soc., 72 (2002), 137-152. 
[4] D. Mosić and D. S. Djordjević, Partial isometries and EP elements in rings with involution, Electron. J. Linear Algebra, 18 (2009), 761-772.

[5] D. Mosić and D. S. Djordjević, Further results on partial isometries and EP elements in rings with involution, Math. Comput. Modelling, 54 (2011), 460465 .

[6] D. Mosić, D. S. Djordjević and J. J. Koliha, EP elements in rings, Linear Algebra Appl., 431 (2009), 527-535.

[7] Y. C. Qu, J. C. Wei and H. Yao, Characterizations of normal elements in rings with involution, Acta. Math. Hungar., 156(2) (2018), 459-464.

[8] Z. C. Xu, R. J. Tang and J. C. Wei, Strongly EP elements in a ring with involution, Filomat, 34(6) (2020), 2101-2107.

[9] S. Z. Xu, J. L. Chen and J. Benítez, EP elements in rings with involution, Bull. Malays. Math. Sci. Soc., 42 (2019), 3409-3426.

[10] R. J. Zhao, H. Yao and J. C. Wei, Characterizations of partial isometries and two special kinds of EP elements, Czechoslovak Math. J., 70(145) (2020), 539551.

[11] R. J. Zhao, H. Yao and J. C. Wei, EP elements and the solutions of equation in rings with involution, Filomat, 32(13) (2018), 4537-4542.

\author{
Dandan Zhao \\ School of Mathematics \\ Yangzhou University \\ 225002 Yangzhou, China \\ e-mail: 1602292980@qq.com \\ Junchao Wei (Corresponding Author) \\ School of Mathematics \\ Yangzhou University \\ 225002 Yangzhou, China \\ e-mail: jcweiyz@126.com
}

